\section{Ökonomische Instrumente für einen umweltverträglichen Verkehr - Machbarkeit und Wirksamkeit}

\author{
von Alexander Eisenkopf, Zeppelin \\ University Friedrichshafen
}

Die Verkehrspolitik ringt seit Jahren um Strategien für einen nachhaltigen bzw. umweltverträglichen Verkehr (sustainable mobility). Hierbei spielt der Einsatz ökonomischer und dabei insbesondere preispolitischer Instrumente eine wichtige Rolle. Der vorliegende Beitrag analysiert den Einsatz solcher Maßnahmen für den Personen- und Güterverkehr auf der Straße. Es wird gezeigt, dass existierende preis- und ordnungspolitische Instrumente bereits in einem hohen Maße zu einer Internalisierung von Externalitäten beitragen. Trotz aller Anstrengungen bestehen aber im Güterverkehr Defizite hinsichtlich einer vollumfänglichen Anlastung des Externalitätenproblems. Die Wirksamkeit preispolitischer Maßnahmen im Hinblick auf die Verkehrsvermeidung oder -verlagerung auf andere Verkehrsträger dürfte allerdings begrenzt sein, solange keine „politischen Preise" festgelegt werden.

\section{Problemstellung}

Seit vielen Jahren ringen die europäische und die nationale Verkehrspolitik um Strategien für einen nachhaltigen bzw. umweltverträglichen Verkehr (,sustainable mobility“). Insbesondere im Güterverkehr scheint das Ziel der Nachhaltigkeit zumindest gefährdet. Hier zeigen sich bereits heute Grenzen der Belastbarkeit von Infrastruktur und Umwelt. Verantwortlich hierfür ist vor allem die starke Zunahme der Transportleistungen im Straßengüterverkehr der letzten Jahre. Aber auch der motorisierte Individualverkehr hat eine Größenordnung erreicht, die bei gegebenen Infrastrukturkapazitäten kaum zu bewältigen ist. Die einschlägigen Prognosen sagen für die nächsten Jahre zudem ein weiteres Verkehrswachstum voraus, das insbesondere vom Güterverkehr getragen wird (Ifmo 2005, S. 32, 60). Ohne geeignete (Gegen-)Maßnahmen scheint damit der „Verkehrsinfarkt" auf den Straßen programmiert und die Belastungsgrenze der Umwelt überschritten.
Vor diesem Hintergrund ist die Forderung nach Konzepten für einen Ressourcen schonenden bzw. umweltverträglichen Güterverkehr verständlich. Allerdings wird der Begriff der Nachhaltigkeit häufig schwammig ge- oder gar für ideologische Zwecke missbraucht. Unstreitig ist, dass Verkehr wie jede andere Produktionsoder Konsumaktivität natürliche und künstliche Ressourcen beansprucht. Dies wäre normalerweise kein Problem, da sich in marktwirtschaftlichen Systemen der Ressourcenverzehr von wirtschaftlichen Aktivitäten in den Preisen widerspiegelt. Verknappungen von Ressourcen bewirken steigende Faktor- und Produktpreise und die Konsumenten sowie Produzenten passen sich dem an. Komplikationen ergeben sich jedoch, wenn es sich um endliche Ressourcen handelt oder mit einer wirtschaftlichen Aktivität „externe Kosten“ verbunden sind.

Beide Sachverhalte werden im Bereich des Verkehrs thematisiert. Transportaktivitäten benötigen Energie, insbesondere in Form von Mineralölprodukten, und beanspruchen damit beschränkt verfügbare Ressourcen. Der Anteil des Verkehrs am gesamten Energieverbrauch in Deutschland ist von 17 Prozent im Jahr 1970 auf heute fast 30 Prozent gestiegen. Innerhalb des Verkehrssektors entfallen 83 Prozent des Energieverbrauchs auf den Straßenverkehr (BMVBW 2005, S. 294 f.). Allerdings hat die Energieeffizienz gerade im Straßengüterverkehr erheblich zugenommen. Der durchschnittliche Kraftstoffverbrauch von schweren Lkw konnte in den letzten 30 Jahren von rund 50 Litern auf ca. 35 Liter je 100 Kilometer gesenkt werden. Nach Einschätzung der Experten ist das Potential für Energieeinsparungen damit noch längst nicht erschöpft.

Ein Problem sind weiterhin die externen Kosten des Verkehrs. Hierunter sind zusätzliche, nicht kalkulierte Lasten für die Allgemeinheit zu verstehen - wie etwa Umweltverschmutzung, Lärmbeeinträchtigungen, ungedeckte Unfallfolgekosten oder Folgen des anthropogenen Klimawandels. Nach einer im Auftrag des internationalen Eisenbahnverbandes UIC erstellten Studie verursachte der Straßenverkehr in Europa im Jahr 2000 ca. 544 Mrd. € an externen Kosten, während die Eisenbahn nur auf gut 12 Mrd. $€$ kommt. Diese Diskrepanz wird allerdings deutlich entschärft, wenn man die erstellten Verkehrsleistungen in die Betrachtung einbezieht. 
So fallen im Güterverkehr auf der Straße $88 € / 1.000$ Tonnenkilometer (Tkm) an externen Kosten an, während die Schiene $18 €$ / $1.000 \mathrm{Tkm}$ verursacht. Der motorisierte Individualverkehr zieht ca. $76 € / 1.000$ Personenkilometer $(\mathrm{Pkm})$ an externen Kosten nach sich, während die Schiene mit $22 € / 1.000$ Pkm eine deutlich günstigere Position einnimmt. Problematisch stellt sich auch die Situation im Personenluftverkehr dar, der mit 52,5 €/1.000 Pkm zwar eine mittlere spezifische Belastungsintensität aufweist, aber mit insgesamt 85 Mrd. $€$ für ca. 16 Prozent der gesamten verkehrsbedingten Externalitäten steht (UIC 2004, S. 72 ff.).

Die Verkehrspolitik ist also aufgefordert, Rahmenbedingungen für einen möglichst umweltverträglichen Verkehr zu setzen. Dabei muss sie die Interdependenzen zwischen Umwelt, Verkehr, Wirtschaftswachstum und Nachhaltigkeit beachten, um nicht der Fiktion zu erliegen, dass Verkehrsvermeidung, Verkehrsverteuerung und Verkehrsverlagerung für sich genommen als sinnvolle Kategorien der Verkehrspolitik anzusehen sind (Willeke 2002). Eine marktwirtschaftlich ausgerichtete Verkehrspolitik sollte sich daher nicht auf dirigistische Maßnahmen oder ideologisch motivierte Regulierungen stützen, sondern innerhalb eines geeigneten ordnungspolitischen Rahmens konsequent ökonomische Instrumente zur Beeinflussung des Verkehrs einsetzen.

Bei der Diskussion um umweltverträglichen Verkehr stehen die Externalitäten des Verkehrs im Vordergrund der Betrachtung. Im Folgenden ist daher zu diskutieren, wie mit ökonomischen Ansätzen die externen Kosten des Verkehrsmittelbetriebes internalisiert werden können. Diese betreffen im Wesentlichen die Schadstoff- und Lärmemissionen, ungedeckte Verkehrsunfallfolgekosten und Kosten des Klimawandels durch $\mathrm{CO}_{2}$-Emissionen sowie verkehrssysteminterne „Stauungskosten“ (Eisenkopf 2002, S. 144). Aus der weiteren Diskussion ausgeklammert wird dagegen das Problem externer Kosten der Verkehrsinfrastruktur selbst (z. B. Bodenversiegelung, Trennungseffekte und Landverbrauch) und die grundsätzliche Problematik nachhaltiger Wachstumsprozesse bei endlichen Ressourcen (Wieland 2002). Die Argumentation wird sich im Wesentlichen auf den Straßenverkehr beziehen, da hier die drängendsten Probleme vermutet werden. Nicht diskutiert werden soll auch die grundsätzliche Frage der Einführung von Benutzungsabgabensystemen zu Finanzierungszwecken im Sinne einer Umstellung der Haushaltsfinanzierung der Verkehrsinfrastruktur auf Nutzerfinanzierung (Wissenschaftlicher Beirat 1997).

\section{2 Ökonomische Instrumente für einen unweltverträglichen Verkehr}

Die ökonomische Theorie hat sich im Rahmen einer mittlerweile sehr ausdifferenzierten Literatur zur Umweltökonomie und Umweltpolitik mit der Wirkungsweise und der Effizienz ökonomischer Instrumente zur Internalisierung externer Effekte beschäftigt. ${ }^{1}$ Generell ist die Umweltpolitik in Deutschland wie auch in vielen anderen Ländern jedoch von ordnungsrechtlichen Maßnahmen bestimmt, die Verbote aussprechen bzw. im Sinne einer Auflagenpolitik bestimmte Standards definieren, deren Missachtung Ordnungsstrafen oder sonstige Geldzahlungen nach sich zieht. Ein Beispiel aus dem Verkehrsbereich wären die Emissionsstandards für Kraftfahrzeuge oder Start- und Landebeschränkungen für zu laute Flugzeuge. Ge- und Verbote sowie Auflagen werden von der ökonomischen Theorie jedoch weder als besonders treffsicher hinsichtlich des Umweltziels noch als effizient im Sinne statischer und dynamischer Effizienzkriterien angesehen (Fritsch, Wein, Ewers 2005, S. 164 ff.).

Theoretisch diskutiert und politisch gefordert wird häufig die Anlastung externer Kosten mittels entsprechender Abgaben (EU-Kommission 1998). Der Grundgedanke dieser auf den Ökonomen A.C. Pigou (1877-1959) zurückgehenden Lösung besteht darin, den Verursacher einer negativen Externalität so zu besteuern, dass es im Optimum zu einer Übereinstimmung von privaten und sozialen Grenzkosten kommt. Man spricht in diesem Zusammenhang auch von einer Preisbildung nach sozialen Marginalkosten. Mit der Anlastung sozialer Zusatzkosten in Form einer Pigou-Steuer würde die Abweichung von den Marginalbedingungen korrigiert und im Modell eine wohlfahrtstheoretisch fundierte Optimallösung realisiert (Fritsch, Wein, Ewers 2005, S. 119 f.). 
Abb. 1: Pigou-Steuer

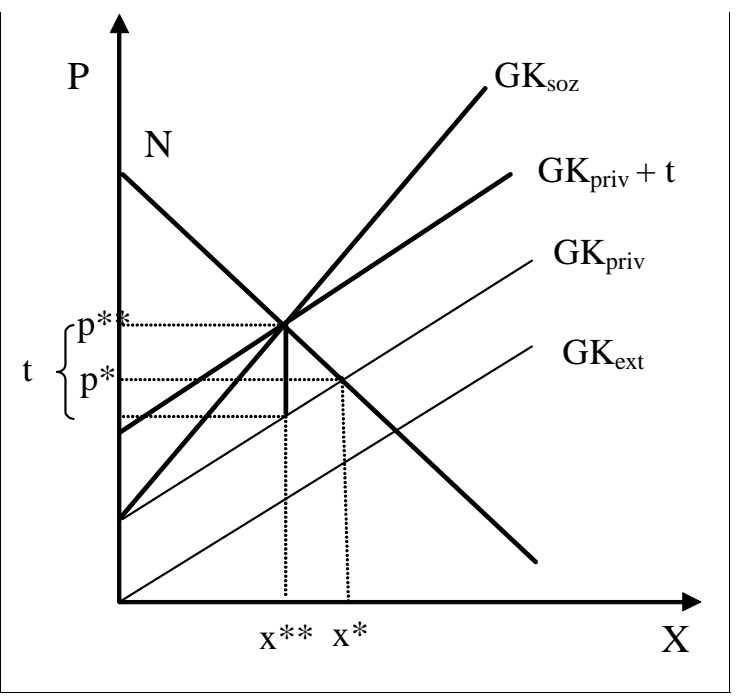

Quelle: eigene Darstellung

In Abbildung 1 würde eine Berücksichtigung lediglich der privaten Grenzkosten $\mathrm{GK}_{\text {priv }} \mathrm{zu}$ einer $\mathrm{zu}$ hohen Ausbringungsmenge $\mathrm{x}^{*}$ bei $\mathrm{zu}$ niedrigem Preis $\mathrm{p}^{*}$ führen. Gesamtwirtschaftlich effizient wäre dagegen die niedrigere Menge $\mathrm{x}^{* *}$ beim Preis $\mathrm{p}^{* *}$, die sich aus dem Schnittpunkt der sozialen Grenzkostenkurve $\mathrm{GK}_{\mathrm{soz}}$ und der Nachfragekurve $\mathrm{N}$ ergibt. Dieses gesellschaftliche Optimum wird erreicht, indem eine auf die Ausbringungsmenge bezogene Steuer $\mathrm{t}$ erhoben wird, die gerade der Differenz zwischen sozialen und privaten Grenzkosten in der Optimalsituation entspricht. Die Steuer verschiebt die private Grenzkostenkurve $\mathrm{GK}_{\text {priv }}$ um $\mathrm{t}$ nach Norden. Angewandt auf den Verkehrsbereich bedeutet dies, dass eine z. B. auf den Fahrzeugkilometer bezogene Anlastung externer Kosten des Straßenverkehrs helfen soll, das soziale Wohlfahrtsoptimum zu erreichen. Die Anlastung der Externalität lässt die Preise für Verkehrsleistungen steigen, während die produzierten Mengen rückläufig sind; zu beachten ist, dass für die Beurteilung der Optimalität einer Lösung die Verwendung des Mittelaufkommens aus der Abgabe irrelevant ist.

Allerdings wird bei der Forderung nach einer Preisbildung zu sozialen Marginalkosten häufig nicht an die gravierenden Informationsprobleme bei der Umsetzung gedacht. Um Allokationsverzerrungen zu vermeiden, müsste die Höhe der (marginalen) sozialen Zusatzkosten in der angestrebten Optimalsituation bekannt sein.
Dies bedingt jedoch die Kenntnis der privaten und gesellschaftlichen Grenzkostenverläufe für jeden Schadensverursacher, d. h. zumindest für typische Verkehrsteilnehmer und typische Verkehrssituationen. Angesichts der vielfältigen Probleme der Erfassung und Bewertung externer Kosten scheint dies selbst für eine (statische) Referenzsituation unmöglich; hinzu kommt das Problem, bei unterschiedlichen, sich überlagernden Schädigungsquellen die externen Effekte eindeutig auf die Verursacher zuzurechnen. Vollends zum Scheitern verurteilt sein dürfte diese Vorgehensweise in einer dynamischen Welt mit sich ständig ändernden Angebots- und Nachfragekonstellationen.

Als praxisnähere Variante zur Pigou-Steuer wurde in der Umweltökonomik der „StandardPreis-Ansatz" entwickelt (Fritsch, Wein, Ewers 2005, S. 123 ff.). Hierbei verzichtet man bewusst darauf, eine Optimalsituation zu erreichen. Es wird vielmehr versucht, über eine Abgabe je Schadenseinheit ein politisch definiertes Vermeidungsziel möglichst effizient zu erreichen. Solange der festgelegte Steuersatz über den Grenzkosten der Schadensvermeidung liegt, werden die Verursacher externer Effekte versuchen, ihre Schadensintensität $\mathrm{zu}$ reduzieren, anstatt die Abgabe zu zahlen. Bei unterschiedlichen Grenzkosten der Schadensvermeidung der Betroffenen kann so - im Gegensatz zu Geboten oder Auflagen - eine effiziente Verringerung des Schadenniveaus im Rahmen der exogen definierten Vorgabe herbeigeführt werden.

Während den bisher besprochenen Internalisierungsansätzen explizit oder implizit das Verursacherprinzip zugrunde liegt, nach dem der Verursacher einer Externalität mit den Konsequenzen seines Tuns oder Unterlassens konfrontiert wird, setzt die Internalisierung durch Verhandlungen auf der Basis des „Coase-Theorems“ an der Idee an, dass es im Grunde keine eindeutige Trennung zwischen Verursachern und Geschädigten gibt. Der Geschädigte hat vielmehr durch seine eigene Disposition dazu beigetragen, dass es zu der Externalität kommt; es handelt sich somit um ein reziprokes Phänomen (Coase 1960).

Nach dem Coase-Theorem ist es möglich, technologisch induzierte externe Effekte auch ohne staatliche Eingriffe durch private Verhandlungen zu internalisieren. Die Aussicht auf Teilhabe an den internalisierungsbedingten 
Wohlfahrtsgewinnen motiviert die Beteiligten zu einer freiwilligen Einigung. Unter bestimmten Annahmen (wohl definierte Eigentumsrechte, Abwesenheit von Transaktionskosten) kommt es durch spontane Koordination zu einer Optimallösung. Eventuell verbleibende Externalitäten sind systemkonform, weil sie aus dem Abgleich der subjektiven Nutzenpositionen der involvierten Wirtschaftssubjekte resultieren. Dies geschieht unabhängig von der Verteilung der „property rights“ bzw. der Haftung in der Ausgangssituation.

Allerdings ist auch die praktische Umsetzung einer Verhandlungslösung nicht unproblematisch. Theoretisch stellt ein solcher Ansatz zwar das beste Internalisierungsverfahren dar, wenn Schadenshaftung des Schädigers gegeben ist. Die Umsetzung scheitert jedoch häufig an prohibitiv hohen Transaktionskosten (Fritsch, Wein, Ewers 2005, S. 131 ff.). Sie versagt insbesondere im Fall großzahliger externer Effekte, wenn diese von vielen Wirtschaftssubjekten verursacht werden und sich auf viele Wirtschaftssubjekte auswirken, wie es z. B. im Verkehrsbereich der Fall ist.

Im Sinne der Überlegungen von Coase ist jedoch darüber nachzudenken, ob die Beschränkung der Aktivitäten eines Verursachers negativer externer Effekte in jedem Fall eine Maximierung der gesellschaftlichen Wohlfahrt beinhaltet. Streng genommen wären die sozialen Kosten einer Aktivität dem privaten Nutzen gegenüberzustellen, denn nur, wenn letztere nicht zur Kompensation ausreichen, ergibt im Sinne von Coase ein Problem. Diese Überlegung läuft auf die Suche nach dem „,cheapest cost avoider" hinaus. Unter Verweis auf die Reziprozität des Externalitätenphänomens muss derjenige bei der Nutzungskonkurrenz um eine knappe Ressource verzichten, welcher dabei die geringsten volkswirtschaftlichen Kosten trägt. Nur wenn die an anderer Stelle anfallenden Nutzenverluste bei der Beschränkung einer negativen Externalität kleiner sind als die vermiedenen Schäden, wäre daher eine solche wirtschaftspolitische Maßnahme sinnvoll (Sälter 1989; Buchanan, Stubblebine 1969). Zu denken wäre hier an die generellen Wachstums- und Beschäftigungseffekte des Verkehrs, die den Externalitäten gegenüberzustellen sind.

Eine weitere Lösungsalternative, welche die Elemente des Standard-Preis-Ansatzes und des Coase-Theorems verknüpft, sind handelbare Schädigungsrechte (Zertifikate). Bei der Zertifikatslösung wird ähnlich wie beim StandardPreis-Ansatz ein gesellschaftlich tolerierbares Maß der Schädigung definiert, welches im Zeitablauf im Sinne der dynamischen Effizienz angepasst werden kann. Für dieses genau definierte Schadensvolumen werden an die Verursacher Schädigungsrechte ausgegeben, die frei handelbar sind. Entsprechend dem sich auf dem Markt für Zertifikate herausbildenden Preis werden die Akteure ihre Handlungen anpassen und entweder Zertifikate behalten bzw. zukaufen, falls ihre Grenzkosten der Schadensvermeidung höher sind als der Preis des Zertifikats, oder im umgekehrten Fall Zertifikate anbieten. Es kommt im Gleichgewicht auf dem Markt für handelbare Schädigungsrechte zu einer statisch effizienten Lösung des Externalitätenproblems. Unter bestimmten Rahmenbedingungen genügt diese Lösung auch dem Ziel der dynamischen Effizienz (Luckenbach 2000, S. 166 f.). Zertifikatslösungen werden derzeit bereits auf Ebene der EU zur Begrenzung der $\mathrm{CO}_{2}$-Emissionen und der Umsetzung des Kioto-Protokolls praktiziert. Die Einbeziehung des Verkehrssektors in einen solchen Emissionshandel wäre grundsätzlich vorstellbar.

\section{Einsatz ökonomischer Instrumente im Personenverkehr}

Umweltverträglichkeit des Verkehrs herzustellen bedeutet aus ökonomischer Perspektive zunächst, den einzelnen Verkehrsaktivitäten ihre negativen Externalitäten anzulasten. Die Anlastung von externen Kosten des Personenverkehrs kann entsprechend der Überlegungen in Abschnitt 2 auf verschiedenem Wege erfolgen, wobei einige der Instrumente bereits heute genutzt werden. ${ }^{2}$ So spielen ordnungsrechtliche Maßnahmen im Personenverkehr eine wichtige Rolle. Beispielhaft seien Abgasemissionsstandards für den Pkw-Verkehr genannt: Entsprechend den einschlägigen Verordnungen der EU wurden in Deutschland Schadstoffgrenzwerte für Pkw je gefahrenen Kilometer festgelegt, die bezüglich der Emissionen von Kohlenmonoxid (CO), Kohlenwasserstoffen und Stickoxiden $\left(\mathrm{HC}\right.$ und $\mathrm{NO}_{\mathrm{x}}$ ) und Partikelemissionen (Dieselfahrzeuge) seit Anfang der 1990er Jahre erhebliche spezifische Emissionsminderungen vor- 
sehen. Hinzu tritt die Differenzierung der KfzSteuer für Pkw nach der Schadstoffklasse und die geplante Einführung von emissionsabhängigen Durchfahrtsbeschränkungen bzw. Fahrverboten in Großstädten, die unter die Begrenzung der so genannten Feinstaub-Richtlinie der EU fallen (Richtlinie 1999/30/EG).

Mit der ab Januar 2005 verbindlichen Norm „Euro-4“ für neu zugelassene Pkw werden selbst nach Einschätzung des Umweltbundesamtes langfristig die Luftreinhaltungsziele in Deutschland und Europa erreicht. Lediglich bei Diesel-Pkw wird hier von den Experten ein weiterer Anpassungsbedarf hinsichtlich der Stickoxide und Partikelemissionen gesehen (Kolke, Friedrich 1998, S. 12 f.)

Auch in anderen Bereichen gibt es staatliche Auflagen bzw. Ge- und Verbote, welche zu einer höheren Umweltverträglichkeit des Personenverkehrs beitragen. Im weitesten Sinne schaffen etwa die Straßenverkehrsordnung und die Straßenverkehrzulassungsordnung einen Regulierungsrahmen, der z. B. den Umfang der von der Gesellschaft zu tragenden ungedeckten Unfallfolgekosten beeinflusst. Wenn Konsens darüber besteht, dass Alkohol am Steuer bzw. überhöhte Geschwindigkeit als häufigste Ursache schwerer Verkehrsunfälle anzusehen sind, dann dürften ein umfassendes Alkoholverbot am Steuer sowie generelle und streckenspezifische Tempolimits mit geeigneter Sanktionsbewehrung geeignete Instrumente zur Reduzierung ungedeckter Unfallfolgekosten sein (SRU 2005). Auch sicherheitsspezifische Zulassungsvorschriften tragen zur Vermeidung von Unfällen und zur Reduzierung von Unfallfolgen bei und senken damit die externen Kosten des PkwVerkehrs. Ähnliches gilt auch für die durch Lärmemissionen verursachten externen Kosten; allerdings finden sich hier auch vielfältige Maßnahmen des passiven Lärmschutzes im infrastrukturellen Bereich (z. B. Lärmschutzwände).

Auch die externen Kosten des Luftverkehrs werden durch ordnungspolitische Maßnahmen beschränkt. An erster Stelle zu nennen sind Nachtflugverbote an bestimmten Flughäfen. Denkbar und zum Teil umgesetzt sind auch Start- und Landeverbote für besonders lärmintensive Flugzeugtypen oder - als preispolitisches Instrument - lärmabhängige Startund Landegebühren.
Es existieren im Personenverkehr also bereits heute wirksame Ansätze zur Internalisierung externer Effekte im Sinne ordnungsrechtlicher Maßnahmen. Ökonomische Internalisierungsansätze im Sinne von fahrleistungsbezogenen Abgaben werden dagegen nicht oder kaum praktiziert, wenn man von der Mineralölsteuer absieht, die indirekt als Instrument zur Anlastung der Wegekosten und externer Kosten des Verkehrsmittelbetriebs angesehen werden kann. Hinzuweisen ist auch auf die emissionsabhängige Kfz-Steuer. Dagegen fehlen alternative Internalisierungsansätze wie z. B. Zertifikate-Lösungen völlig. Es stellt sich daher die Frage, ob in Zukunft zusätzliche preispolitische Maßnahmen (oder andere Instrumente) zur Internalisierung von Externalitäten erforderlich sind.

Analysiert man die aktuell vorgelegten Schätzungen für die externen Kosten des PkwVerkehrs in Europa, zeigt sich, dass die Unfallfolgekosten mit über 40 Prozent die externen Kosten des Pkw- Verkehrs in Europa dominieren. Weitere 23 Prozent entfallen auf den Klimawandel und weitere 17 Prozent auf Luftverschmutzung durch Schadstoffemissionen (UIC 2004, S. 72). Bedeutet nun Internalisierung dieser Externalitäten eine Umlage der errechneten Kostensummen auf die geleisteten Personenkilometer? Entsprechend der von der UIC vorgelegten Berechnungen müssten dann für die genannten drei Kostenkategorien 61 Euro je 1.000 Pkm angelastet werden. Bei einem für Deutschland repräsentativen Besetzungsgrad von ca. 1,5 Personen je Pkw würde dies eine Belastung von ca. 41 Cent je Pkw-Kilometer bedeuten.

Eine solche „Umlegung der externen Kosten" auf die Nutzer ist aus verschiedenen Gründen fragwürdig. Zum einen lassen sich durchaus kritische Anmerkungen im Hinblick auf die von der UIC vorgelegten Kostenschätzungen machen. So werden in die Unfallfolgekostenschätzungen nicht nur medizinische Kosten und Ressourcen-Ausfallkosten einkalkuliert, sondern auch Wohlfahrtsverluste für Unfalltote und Verletzte, deren Kalkulation auf „Zahlungsbereitschaftsansätzen“ beruhen (,willingness to pay“ $<$ WTP $>$ ) und damit merklich überhöhte Kosten ausweisen. Auch die Berechnung anderer externer Kostenkategorien basiert mit auf WTPAnsätzen. Hinsichtlich der durch $\mathrm{CO}_{2}$ verursachten Kosten des Klimawandels wird von einem sehr anspruchsvollen Vermeidungsziel 
(minus $50 \%$ bis zum Jahre 2030) und einem daraus resultierenden Schattenpreis von $140 €$ / $\mathrm{t}$ $\mathrm{CO}_{2}$ ausgegangen (UIC 2004, S. 23 ff.). Insgesamt ist die Höhe der in der Diskussion befindlichen Kostenschätzungen daher durchaus in Frage zu stellen.

Hinzu kommt, dass Anlastung externer Kosten im Sinne einer Pigou-Steuer nicht bedeuten kann, die errechneten absoluten Kosten in einer Durchschnittsbetrachtung auf die Nutzer umzulegen. Erforderlich wären vielmehr Informationen über die jeweiligen externen Grenzkosten. Auch diesbezüglich existieren mittlerweile Abschätzungen, deren Validierung und praktische Umsetzung allerdings noch aussteht (Kossak 2004, S. 537 f.).

Bei vorsichtiger Abschätzung kann man zu dem Ergebnis kommen, dass bei einer zukunftsorientierten Betrachtung eine zusätzliche Abgabe zur Anlastung externer Kosten des PkwVerkehrs nicht erforderlich ist. Bezüglich der Schadstoffemissionen sei auf die absehbaren Emissionsminderungen hingewiesen, die sich mit der in Zukunft größeren Verbreitung emissionsarmer Fahrzeuge in der Pkw-Population ergeben werden (Kolke, Friedrich 1998). Auch bezüglich der externen Lärmkosten dürften aktive und passive Lärmschutzmaßnahmen eine wichtige Rolle spielen, die Konsequenzen verkehrsbedingter Lärmemissionen auf ein akzeptables Maß zu senken (Puwein 2005), zumal hier methodische Probleme im Hinblick auf die Anlastung durch eine Gebühr bestehen (Reinhold 1998). Hinsichtlich ungedeckter Unfallkosten ist grundsätzlich zu hinterfragen, ob eine kilometerbezogene Abgabe das geeignete Anlastungsinstrument für risikobehaftete Schadensereignisse ist; im Vordergrund der Überlegungen sollte die Kompensation verbleibender Kosten über das Versicherungssystem stehen. Außerdem dürfte ein Großteil der ungedeckten Unfallkosten eher auf Staatsversagen denn auf Marktversagen beruhen, weil der Staat es versäumt, geeignete ordnungsrechtliche Maßnahmen zur Unfallvermeidung zu definieren und durch ungenügenden Infrastrukturausbau mit $\mathrm{zu}$ einer Steigerung der Unfallzahlen beiträgt.

Auch im Hinblick auf die Wegekostendeckung des Pkw-Verkehrs dürfte kaum ein Bedarf für eine zusätzliche preispolitische Maßnahmen bestehen. Wie die letzte vorliegende Studie zur Wegekostendeckung des DIW mit
Bezugsjahr 1997 ausweist, erreichten inländische Personenkraftwagen eine Wegekostendeckung von insgesamt 218 Prozent und auf Bundesfernstraßen sogar von 349 Prozent (Link, Rieke, Schmied 2000, S. 68). Seither wurde die Mineralölsteuerbelastung der Pkw durch die Ökosteuer deutlich erhöht (von 0,50 auf 0,67 Cent/l Vergaserkraftstoff), so dass die Wegekostendeckungsgrade sich zumindest nicht verschlechtert haben dürften. Bei einem Durchschnittsverbrauch von 8,4 l/ 100km für Pkw mit Otto-Motor und 6,9 l/ $100 \mathrm{~km}$ für Dieselfahrzeuge errechnet sich eine Abgabenleistung von 5,6 Cent je Fahrzeugkilometer für Pkw mit Otto-Motor und 3,3 Cent für DieselPkw. Damit wird - auch im Sinne der als Ökosteuer adressierten Mineralölsteuererhöhung ein wesentlicher Beitrag zur Anlastung der externen Kosten von $\mathrm{CO}_{2}$-Emissionen erbracht.

Als Problem verbleiben die externen Stauungskosten des Pkw-Verkehrs. Stauungskosten wären nur über zeit- und verkehrsflussabhängige Stauungsabgaben sinnvoll anzulasten. Ungeachtet der generellen Diskussion um die Einführung einer Pkw-Maut auf Autobahnen zu Finanzierungszwecken bleibt die Einführung belastungsabhängiger Straßenbenutzungsgebühren aus ökonomischer Sicht wünschenswert, um die erheblichen volkswirtschaftlichen Schäden von Verkehrsstaus zu vermeiden. Auch hier stellt sich jedoch das Problem der Operationalisierung entsprechender Grenzkostenpreise und der Umsetzung in der Praxis.

\section{Einsatz ökonomischer Instrumente im Güterverkehr}

Analog zu den für den Personenverkehr beschriebenen ordnungsrechtlichen Maßnahmen existieren auch für den Güterverkehr Verbote, Gebote und Auflagen, die auf eine Reduzierung volkswirtschaftlich relevanter Externalitäten hinwirken. Auch hier kommt es durch die sukzessive Verschärfung der spezifischen Abgasgrenzwerte je Kilowatt Motorleistung zu einer deutlichen Reduzierung des Schadstoffemissionsproblems. Mit Einführung der Abgasnorm Euro-5 werden die spezifischen Emissionen des Lkw gegenüber Euro-0 im Mittel um 90 Prozent reduziert. Aufgrund der schnelleren Verbreitung von schadstoffarmen Fahrzeugen in der gewerblich genutzten Fahrzeugpopulation dürften sich 
die mit schärferen Standards ergebenden Emissionsminderungen trotz der kontinuierlich wachsenden Verkehrs- und Fahrleistungen eher einstellen als im Personenverkehr. Derzeit ist der Nettoeffekt der Grenzwertverschärfung eindeutig positiv für die Umwelt: Gegenüber dem Jahr 2000 konnten die tatsächlichen Schadstoffemissionen des Straßengüterverkehrs trotz steigender Fahrleistungen im Mittel um 40 Prozent gesenkt werden (BGL 2006, S. 45).

Auch hinsichtlich der Anlastung externer Kosten im Güterverkehr gelten die im Hinblick auf die Kostenberechnungen formulierten generellen kritischen Anmerkungen. Die externen Kosten des Güterverkehrs werden laut der für die UIC erstellten Studie durch Schadstoffemissionen (49\%) und Kosten des $\mathrm{CO}_{2}$-bedingten Klimawandels (19\%) dominiert. Problematisiert wird in der öffentlichen Diskussion insbesondere die Entwicklung der zukünftigen $\mathrm{CO}_{2}$-Emissionen des Güterverkehrs (Hopf, Voigt 2004; SRU 2005). Angesichts der derzeit vorliegenden Grenzwerte und der weiter absehbaren Verschärfungen der Emissionsstandards ist aber die Notwendigkeit der Einführung zusätzlicher fahrleistungsbezogener Emissionsabgaben mit Fragezeichen zu versehen. Hinsichtlich der Höhe der externen Kosten der $\mathrm{CO}_{2}$-Emissionen sei auch auf das zum Personenverkehr Angeführte verwiesen. Hier wäre allerdings für den Güterverkehr eine Teilnahme am allgemeinen $\mathrm{CO}_{2}{ }^{-}$ Emissionshandel denkbar. Die relativ geringe Zahl gewerblicher Transportunternehmen macht eine Einbeziehung bei realistischen Transaktionskosten durchaus denkbar. Hinzuweisen ist auch darauf, dass es sich bei den $\mathrm{CO}_{2}$-Emissionen um ein globales Problem handelt, das nicht mit branchenbezogenen Lösungsansätzen angegangen werden sollte (Puwein 2005).

Im Gegensatz zum Personenverkehr muss sich der Straßengüterverkehr traditionell auch das Argument ungedeckter Infrastrukturkosten zurechnen lassen. Laut den Berechnungen des DIW lag der Wegekostendeckungsgrad inländischer Lkw im Jahre 1997 nur bei knapp 76 Prozent (Link, Rieke, Schmied 2000, S. 68). Mit der Einführung der Ökosteuer dürfte sich allerdings auch hier der Beitrag zu den vom Schwerverkehr verursachten Wegekosten verbessert haben, wenn auch die gegenüber anderen EUStaaten sehr hohe Steuerbelastung viele Unternehmen dazu motiviert, verstärkt im Ausland zu tanken. Die Wegekostenproblematik schwerer Lkw über zwölf Tonnen auf Autobahnen ist in jedem Fall durch die Einführung der Lkw-Maut zum 1.1.2005 abschließend gelöst, so dass für diesen bedeutenden Teilmarkt die zu zahlende Mineralölsteuer ausschließlich als Anlastung von negativen Externalitäten zu bewerten ist.

Hinzuweisen ist auch darauf, dass Umweltbeeinträchtigungen durch den Güterverkehr im Wesentlichen an die erbrachten Fahrleistungen gekoppelt sind. Mit einer Entkopplung von Verkehrs- und Fahrleistungen lassen sich daher höhere Verkehrsleistungen ohne zusätzliche Umweltbeeinträchtigungen realisieren. In der Vergangenheit war dieser Entkopplungsmechanismus durchaus fruchtbar. So stiegen die Verkehrsleistungen des Straßengüterverkehrs in Deutschland von 1991 bis 2004 um 55 Prozent, während die Fahrleistungen in diesem Zeitraum um 42 Prozent zunahmen. Wenn sich auch in Zukunft eine weitere Entkopplung von Verkehrs- und Fahrleistungen realisieren lässt, was durchaus im ökonomischen Eigeninteresse der Straßengüterverkehrsbranche liegt, dürfte sich die Umweltbilanz des Straßengüterverkehrs auch ohne weitere staatliche Eingriffe verbessern.

Insgesamt gestaltet sich eine Bewertung der Umweltverträglichkeit des Straßengüterverkehrs trotz aller Fortschritte im Detail weniger eindeutig als im Personenverkehr. Angesichts der erheblichen Wachstumsdynamik der Branche spricht einiges dafür, die Emissionsstandards für Nutzfahrzeuge weiter zu verschärfen. Auch die Sicherheits- und Sozialvorschriften bzw. deren Einhaltung sind ein Thema im Hinblick auf ungedeckte Unfallfolgekosten. Eine zusätzliche Emissionsabgabe scheint dagegen aus ökonomischer Sicht entbehrlich, zumal es bei der Internalisierung externer Effekte nicht um eine Art Schadensersatz für die Betroffenen geht, der durch vollständige Anlastung der berechneten Schadenssumme herzustellen ist (wie bei den Infrastrukturkosten), sondern um die Steuerung der verkehrsbezogenen Aktivitäten hin zu einem „gesamtwirtschaftlich effizienten Schädigungsniveau“. Hinsichtlich des Problems der $\mathrm{CO}_{2}$ Emissionen bleibt die Einbeziehung des Güterverkehrs insgesamt (also auch des Schienenverkehrs und der Binnenschifffahrt) in den europäischen Emissionshandelsmechanismus eine aus 
ökonomischen Effizienzerwägungen durchaus plausible Alternative.

\section{Zum Spannungsverhältnis von Verkehrs- wachstum, Mobilität und Nachhaltigkeit}

In der Gesellschaft wächst die Skepsis gegenüber dem Verkehr und seinen Umweltwirkungen. Bei nüchterner ökonomischer Betrachtung stellt jedoch die Vermeidung oder Verlagerung von Verkehren keinen Eigenwert dar. Solange eine plausible und realistische Anlastung externer Kosten erfolgt, sollte der Staat sich aus den Entscheidungen der Marktteilnehmer über die Nutzung der Verkehrsträger und damit der Steuerung des „Modal-Split“ heraushalten. ${ }^{3}$

Insbesondere ist das Verkehrsmittel mit den absolut niedrigsten externen Kosten nicht unbedingt das volkswirtschaftlich günstigste Instrument zur Lösung einer Transportaufgabe. Ein Transport, der wegen der Nutzung der Bahn mehrfach umgeschlagen werden muss, bedeutet für den Kunden in der Regel einen höheren Aufwand. Außerdem verlängert sich meistens die Beförderungsdauer. Beklagt wird von den „Verladern“ häufig auch die schlechte Qualität und mangelnde Pünktlichkeit von Bahntransporten - insbesondere bei internationalen Verkehren. Eine isolierte Verlagerungsstrategie von der Straße auf die Schiene, um externe Kosten zu reduzieren, führt daher möglicherweise zu erheblichen Nutzeneinbußen für die Volkswirtschaft insgesamt.

Tragfähige Konzepte für einen Ressourcen schonenden Güterverkehr müssen zudem den Einfluss der generellen politischen Rahmenbedingungen beachten. Ein Großteil des Verkehrszuwachses in den letzten zehn Jahren beruht auf der Vollendung des europäischen Binnenmarktes und der Integration der europäischen Volkswirtschaften. Auch das für die Zukunft prognostizierte Wachstum der Gütertransporte resultiert schwerpunktmäßig aus der Erweiterung der EU nach Osten (Acatech 2006). Wenn die wirtschaftliche Integration und Erweiterung der Europäischen Union auch wegen der wohlfahrtssteigernden Effekte - politisch gewünscht wird, ist zusätzlicher Verkehr unausweichlich. Es stellt sich allerdings die Frage, wie dieser möglichst umweltverträglich gestaltet werden kann.
Eine wichtige Rolle in diesem Kontext spielt zweifelsohne der Schienenverkehr - vor allem wegen der großen Transportentfernungen in Europa, die den Einsatz der Eisenbahn oder des kombinierten Verkehrs Schiene/Straße (bzw. Binnenschiff / Straße) erst rentabel machen. Tatsächlich tun sich die Eisenbahnen im Wachstumssegment des internationalen Güterverkehrs immer noch sehr schwer. Viele technische und organisatorische Hindernisse machen den Schienenverkehr zum einzigen europäischen Markt, der sich durch nach wie vor wirksame Binnengrenzen auszeichnet.

Die EU-Kommission hat diese Probleme erkannt und unternimmt seit geraumer Zeit erhebliche Anstrengungen im Hinblick auf entsprechende Reformen. Sie hat in ihrer Halbzeitbilanz zum Weißbuch Verkehrspolitik festgestellt, dass für die Zukunft der Eisenbahnen weitere Effizienzsteigerungen unverzichtbar sind. Nur so können diese in Zukunft den Anforderungen der Wirtschaft an logistische Leistungsqualitäten entsprechen. Gefordert wird eine konsequente Öffnung der Netze für mehr Wettbewerb und eine Modernisierung der Strukturen im Bahnsektor. Dies möchte die EU-Kommission durch verschiedene Maßnahmenpakete beschleunigen. Sie beinhalten insbesondere die Gewährung von Zugangsrechten zu den nationalen Schienengüterverkehrsmärkten für neue Anbieter und die Harmonisierung von technischen Standards und Sicherheitsvorschriften (Scherp 2005).

Im Rahmen eines Konzepts für umweltverträglichen Güterverkehr muss aber auch der Straßentransport weiterentwickelt werden. Nicht adäquat erscheinen Maßnahmen, die allein auf die Eindämmung des Straßengüterverkehrs zielen, denn Lkws werden auch in Zukunft den größten Teil der Verkehrsbelastung zu bewältigen haben. Zur Sicherung der Nachhaltigkeit sind dagegen insbesondere Auflagen in Form von Abgasnormen oder Sicherheits- und Sozialvorschriften geeignet, wie sie heute bereits wirksam eingesetzt werden. Auch preispolitische Maßnahmen sind grundsätzlich marktwirtschaftskonform und aus der Perspektive der Nachhaltigkeit wünschenswert, wenn sie in korrekter Weise das Ausmaß der noch nicht internalisierten Umweltschäden oder die Knappheit endlicher Ressourcen widerspiegeln. 
Hier bestehen allerdings derzeit noch erhebliche Unsicherheiten hinsichtlich einer korrekten Anlastung. Beabsichtigte Eingriffe in die Preisbildung stützen sich häufig auf ,politische Preise“, welche in der politischen Agenda mit dem Schutz der Bahnen vor unfairen Wettbewerbsbedingungen und ihrer Ertüchtigung zur Lösung der Wachstumsprobleme der Verkehrsmärkte in Zusammenhang gebracht werden. So haben die Deutsche Bahn und die französische Staatsbahn eine Untersuchung bei der Unternehmensberatung McKinsey in Auftrag gegeben, die sich mit den Verlagerungswirkungen einer auf das Schweizer Niveau erhöhten LkwMaut in Europa beschäftigt. McKinsey kommt zu dem Ergebnis, dass selbst eine Lkw-Maut in der Größenordnung von 49 Cent je Fahrzeugkilometer den Marktanteil der Bahnen in Europa von derzeit 14 Prozent nur um zwei bis drei Prozentpunkte erhöhen könnte (McKinsey 2005). Die Gutachter gehen allerdings davon aus, dass bei einer Beibehaltung des Staus quo in Europa die Modal-Split-Anteile der Bahnen auf unter zehn Prozent abnehmen werden.

Eine im Gegenzug durch die „International Road Transport Union“ (IRU) und den Bundesverband Güterkraftverkehr, Logistik und Entsorgung e.V. (BGL) in Auftrag gegebene Studie kommt zu dem Ergebnis, dass selbst bei einer Maut von 1 Euro je Fahrzeugkilometer nur ca. ein Prozent des Straßengüterverkehrsvolumens auf die Schiene verlagert werden würde - und dies bei einer volkswirtschaftlichen Zusatzbelastung von 170 Mrd. Euro in Europa (Transcare 2006). Gebühren in dieser Größenordnung wären jedoch weitgehend willkürlich und durch wissenschaftliche Berechnungen nicht mehr zu begründen. Damit dürften Strategien für einen umweltverträglichen Güterverkehr, die allein auf einer Verteuerung des Gütertransports durch Lkw basieren, zum Scheitern verurteilt sein.

Selbst wenn man das von den Bahnenvertretern propagierte Szenario für realistisch hält, zeigt sich eine erhebliche Diskrepanz zwischen den notwendigen willkürlichen Eingriffen in die Preisbildung und dem erzielbaren Verlagerungseffekt. Dieser Befund wird gestützt durch die Erfahrungen in der Schweiz, Frankreich und in Deutschland, wo durch die Einführung von Lkw-Mauten kein signifikanter Einfuß auf das Wachstum des Straßengüterverkehrs zu beobachten war.
Gefordert ist daher ein umfassender Ansatz, der die Stärken und Schwächen der jeweiligen Verkehrssysteme berücksichtigt. Straße und Schiene sollten nicht gegeneinander ausgespielt werden, sondern müssen sich ergänzen. Wenn die Schiene einen größeren Anteil am Verkehrswachstum übernehmen soll, muss ihre Wettbewerbsfähigkeit vor allem durch interne Umstrukturierungen und die Öffnung der Märkte gestärkt werden. Aber auch der weitere Ausbau der Infrastruktur, insbesondere des stark belasteten Straßensektors stärkt die Umweltverträglichkeit des Verkehrs, da hierdurch Staus und Unfälle reduziert werden.

\section{Anmerkungen}

1) Einen guten Überblick dazu geben Fees (1998) oder Fritsch, Wein und Ewers (2005).

2) Einen Überblick über Instrumente im Verkehr liefert Puwein 2005.

3) „Modal-Split“ wird in der Verkehrsstatistik die Verteilung eines Transportaufkommens auf verschiedene Verkehrsträger („Modi“) genannt (Anm. d. Red.).

\section{Literatur}

Acatech, 2006: Mobilität 2020, Perspektiven für den Verkehr von morgen. München, Berlin

BMVBW - Bundesministerium für Verkehr, Bau und Wohnungswesen, 2005: Verkehr in Zahlen 2005/2006. Hamburg

Buchanan, J.M.; Stubblebine, W.C., 1969: Externality. In: Arrow, K.J.; Scitovsky, T.: Readings in Welfare Economics. London: Allen \& Unwin

BGL - Bundesverband Güterkraftverkehr, Logistik und Entsorgung, 2006: Jahresbericht 2005/2006. Frankfurt a. M.

Coase, R.H., 1960: The Problem of Social Cost. In: The Journal of Law and Economics 3 (1960), S. 1-44

Eisenkopf, A., 2002: Effiziente Straßenbenutzungsabgaben. Theoretische Grundlagen und konzeptionelle Vorschläge für ein Infrastrukturabgabensystem. Hamburg: Deutscher Verkehrs-Verlag

EU-Kommission, 1998: Faire Preise für die Infrastrukturbenutzung: Ein abgestuftes Konzept für einen Gemeinschaftsrahmen für Verkehrs-Infrastrukturgebühren in der EU. Brüssel (Weißbuch KOM (1998) 466 endg.)

Fees, E., 1998: Umweltökonomie und Umweltpolitik. München: Vahlen 
Fritsch, M.; Wein, Th.; Ewers, H.-J., 2005: Marktversagen und Wirtschaftspolitik. Mikroökonomische Grundlagen staatlichen Handelns. München: Vahlen

Hopf, R.; Voigt, U., 2004: Verkehr, Energieverbrauch, Nachhaltigkeit. Heidelberg: PhysicaVerlag

Ifmo - Institut für Mobilitätsforschung, 2005: Zukunft der Mobilität. Szenarien für das Jahr 2025. Erste Fortschreibung. Berlin

Kolke, R.; Friedrich, A., 1998: Gegenüberstellung von Pkw mit Verbrennungskraftmaschinen, Hybridantrieben und Brennstoffzellen aus Umweltsicht. Berlin (VDI-Berichte Nr. 1418)

Kossak, A., 2004: Straßenbenutzungsgebühren. Mittel der Stauminderung und Verkehrslenkung. In: Internationales Verkehrswesen 56 (2004), S. 536-540

Link, H.; Rieke, H.; Schmied, M., 2000: Wegekosten und Wegekostendeckung des Straßen- und Schienenverkehrs in Deutschland im Jahre 1997. Gutachten des Deutschen Instituts für Wirtschaftsforschung im Auftrag des Bundesverbandes Güterkraftverkehr, Logistik und Entsorgung und des ADAC. Berlin

Luckenbach, H., 2000: Theoretische Grundlagen der Wirtschaftspolitik. München: Vahlen

McKinsey \& Comp., 2005: The Future of Rail Freight in Europe. A Perspective on the Sustainability of Rail Freight in Europe. Brussels

Puwein, W., 2005: Verkehrspolitische Instrumente für einen nachhaltigen Kraftfahrzeugverkehr. In: WIFO Monatsberichte Nr. 12 (2005), S. 851-867

Reinhold, T., 1998: Die Internalisierung externer Kosten des Verkehrslärms. In: Zeitschrift für Verkehrswissenschaft 69 (1998), S. 245-270

Sälter, P.M., 1989: Externe Effekte: „Marktversagen” oder Systemmerkmal. Heidelberg: PhysicaVerlag

Scherp, J., 2005: Railway (De)Regulation in EU Member States and the Future of European Rail. In: CES-ifo DICE-Report 4, S. 26-33

SRU - Sachverständigenrat für Umweltfragen, 2005: Umwelt und Straßenverkehr. Hohe Mobilität - Umweltverträglicher Verkehr. Berlin

Transcare AG, 2006: Einfluss der Lkw-Maut auf den Modal Split im Güterverkehr. Wiesbaden

UIC - Union Internationale des Chemins de Fer, 2004: External Costs of Transport. Zürich, Karlsruhe; Oktober 2004

Wieland, B., 2002: Economical and Ecological Sustainability - The Identity of Opposites. Dresden (Diskussionsbeiträge aus dem Institut für Wirtschaft und Verkehr der Fakultät Verkehrswissenschaften „Friedrich List“ an der Technischen Universität Dresden, Nr. 2)
Willeke, R., 2002: Nachhaltigkeit durch Wachstum. Köln: Deutscher Instituts-Verlag

Wissenschaftlicher Beirat beim Bundesminister für Verkehr, 1997: Neue Wege zur Finanzierung und Nutzungsoptimierung für die Straßeninfrastruktur. In: Zeitschrift für Verkehrswissenschaft 68 (1997), S. 73-93

\section{Kontakt}

Prof. Dr. Alexander Eisenkopf

Lehrstuhl für Allgemeine Betriebswirtschaftslehre und Mobility Management

Zeppelin University gGmbH 88045 Friedrichshafen

Email: aeisenkopf@zeppelin-university.de 